\title{
3. NEUTRON BEAM ANALYTICAL METHODS
}

$\mathrm{T}$ he neutron was discovered by James Chadwick in 1932. It is a sub-atomic particle found in the nuclei of all atoms heavier than hydrogen. It has zero electric charge and is slightly heavier than the proton. Neutrons bound inside stable nuclei are themselves stable. However, a free neutron is unstable and will $\beta$-decay into a proton, emitting an electron and an antineutrino with a half-life of 10.23 minutes.

$$
n^{0} \rightarrow p^{+}+e^{-}+\bar{v}_{e}
$$

Soon after its discovery, scientists realised that beams of free neutrons could be used as probes to explore properties of matter. Due to its zero electric charge, the neutron can penetrate deep into matter and can provide information through a variety of neutron capture or scattering interactions [90].

\subsection{BASIC PRINCIPLES OF NEUTRON BEAM ANALYSIS}

Analytical neutron techniques require sources with constant and sufficiently high neutron fluxes, and suitable neutron energies. Isotopic sources are commonly used for small-scale laboratory studies. The radioisotope ${ }^{252} \mathrm{Cf}$ produces neutrons directly in its decay. Other sources combine a-emitting radioisotopes with a ${ }^{9} \mathrm{Be}$ target. Neutrons are released when the beryllium disintegrates under a-bombardment. Common sources of this type are ${ }^{210} \mathrm{Po}-{ }^{9} \mathrm{Be},{ }^{239} \mathrm{Pu}-{ }^{9} \mathrm{Be}$ and ${ }^{241} \mathrm{Am}-{ }^{9} \mathrm{Be}$. However, the neutron flux provided by isotopic sources tends to be small.

For detailed studies scientists require much more intensive neutron fields from which neutron beams may be extracted. Such fields may be produced in nuclear reactors (also called research reactors), through the fission of nuclear fuel such as ${ }^{235} \mathrm{U}$. A constant and intense neutron flux is produced by a controlled and sustained chain reaction in the reactor. $A$ large number of research reactors have been built all over the World. Many of them (e.g. those in Garching, Delft, Saclay, Budapest and Řež near Prague) still provide very reliable and effective neutron fields for research [91].
In addition to fission reactors, neutron generators can produce $14 \mathrm{MeV}$ fast neutrons, using a beam of deuterons on a tritium target, through the ${ }^{3} \mathrm{H}(\mathrm{d}, \mathrm{n})^{4} \mathrm{He}$ reaction. Fast neutrons with varying energy in the range from 20 to 35 $\mathrm{MeV}$ can also be produced using several nuclear reactions using accelerated protons and deuterons with deuterium, beryllium and lithium targets [92].

Another large scale neutron source is the spallation source, where a high energy accelerated proton beam hits a heavy metal (e.g. Tungsten) target. As a result intense neutron radiation is emitted. The largest spallation sources used for research purposes in Europe are ISIS in the UK and SINQ in Switzerland. Both fission reactors and spallation sources are very large, expensive facilities, but they serve scientific aims in a very effective way [93].

The most common questions addressed by neutron beam analysis are the provenance of objects (i.e. the origin of their raw material), or the workshop or technique used in its production. Sometimes, it is important to know whether the object is an original or counterfeit. In many cases these questions can be answered using non-destructive analytical methods.

There is a large variety of neutron techniques, based on different types of interaction with matter, which offer a wide range of methods to explore the composition or structural features of samples. The bulk elemental composition of objects can be determined from the detection of characteristic $\gamma$-rays produced in $(n, \gamma)$ reactions. Investigation of elastic or inelastic scattering patterns can provide information on the atomic, molecular or nano-scale structural properties including their crystalline or amorphous morphology, phase composition, mechanical strains, impurities, etc. Neutrons can also be used to screen objects whose inner structure is unknown. Using neutron techniques, $2 \mathrm{D}$ or $3 \mathrm{D}$ images of objects can be reconstructed in a manner similar to X-ray radiography or tomography [94].

\section{NEUTRON ACTIVATION ANALYSIS (NAA)}

Neutron activation analysis is a multi-element analytical technique used for qualitative and quantitative analysis of major, minor, and trace elements. Samples with masses 
typically in the range from $\mathrm{mg}$ to hundreds of $\mathrm{mg}$ are irradiated with neutrons. This results in the formation of radioisotopes from isotopes of elements present in the samples, mostly via the $(n, y)$ nuclear reaction with thermal neutrons (neutron radiative capture).

The newly formed radioisotopes decay by particle emission or, more importantly, by emitting $\mathrm{Y}$-rays characteristic of the radioisotopes produced in the capture reaction. The irradiation is usually carried out in a nuclear reactor but other neutron sources (radioisotopic or accelerator based) can also be used. The neutrons used for irradiation are categorised as cold, thermal, epithermal (resonance) or fast, according to their energy.

In general, the lower the neutron energy, the higher the probability of the $(n, y)$ nuclear reaction. Detection limits are primarily determined by neutron capture cross-sections, i.e. the probability of the $(n, y)$ reaction.

Thermal neutron capture cross-sections can vary over seven orders of magnitude. Those elements with the largest neutron capture cross-sections can be measured with the lowest detection limits. These are some rare-earth elements, namely $\mathrm{Eu}, \mathrm{Dy}, \mathrm{Ho}, \mathrm{Sm}, \mathrm{Lu}$, as well as $\mathrm{Mn}$, In, Re, Ir and Au. On the other hand, elements that are hard to detect are those with low neutron capture cross-sections. These include Si, S and Fe. The element $\mathrm{Pb}$ cannot usually be determined at all, because it only forms a short-lived ${ }^{207} \mathrm{~Pb}$ radionuclide with a half-life of $0.8 \mathrm{~s}$.

The irradiation time and the time gap between irradiation and the subsequent $y$-ray measurements are chosen to optimise detection limits for the elements of interest. Commonly $y$-rays are detected and analysed using spectrometers with a High Purity Germanium (HPGe) semi-conductor detector. The evaluation of the spectra is performed offline using $Y$-ray spectroscopy computer programs.

In general, the energies of detected $y$-rays identify isotopes of particular elements in the sample and their intensities are proportional to the element concentration. The analyses are usually calibrated and quantified against appropriate element standards, irradiated and measured under the same conditions or using the $k_{0}$-standardisation process, in which neutron flux monitors are used.

This mode, which uses a purely instrumental, nondestructive, approach is commonly called instrumental neutron activation analysis (INAA). Sometimes the sample is subjected to chemical separation in which case an element of interest is selected before neutron irradiation (pre-separation NAA, PS-NAA), or a radiochemical separation of particular radioisotopes is carried out from the irradiated sample (radiochemical NAA, RNAA). In this way, it is possible to remove undesirable radionuclides and to improve substantially the detection limits for selected elements. NAA can detect up to 74 elements depending on the experimental procedure, with minimum detection limits ranging from $10^{-7} \mathrm{~g} / \mathrm{g}$ to $10^{-12} \mathrm{~g} / \mathrm{g}$, depending on the elements and matrix composition.
The NAA technique requires a small sample to be taken from the object analysed, (e.g. by drilling in an inconspicuous place) but the size of the sample is usually so small that damage to the object is minimised. Due to its high potential for accuracy and well defined theoretical background (all sources of uncertainty can be experimentally evaluated or modeled), NAA with relative standardisation has recently been recognised as a primary method of measurement, e.g. a method with the highest metrological properties.

A widespread INAA technique has been developed based on work by George Hevesy in 1936. In this case, an encapsulated sample, about $50 \mathrm{mg}$, is positioned inside the core of a research reactor and irradiated for a known period of time. Following a necessary cooling time, the so-called delayed or decay radiation is counted, usually by $\gamma$-spectrometry. Several $\gamma$-spectroscopy measurements may be performed a few minutes, days, or weeks after irradiation in order to detect the short-, medium- and long-lived products, respectively. Since the neutron flux in the reactor core is typically $10^{12}-10^{14} \mathrm{~cm}^{-2} \mathrm{~s}^{-1}$, the method is very sensitive for a series of trace elements. However, because of the high neutron flux, there is a risk of significant radiation damage and of producing nuclides with long half-lives in the sample [95].

\section{PROMPT GAMMA ACTIVATION ANALYSIS}

A slightly different technique is prompt gamma activation analysis (PGAA or PGNAA). In this case even large-sized samples can be placed in a guided neutron beam extracted from a reactor. Due to their low energy and relatively low intensity $\left(10^{7}-10^{9} \mathrm{~cm}^{-2} \mathrm{~s}^{-1}\right)$ guided neutron beams are perfect tools to study valuable and irreplaceable cultural heritage objects and artefacts, where destructive or invasive methods are out of the question. Generally neutron investigations cause no observable damage to artefacts and most of the induced radioactivity decays within a few days.

When the sample is irradiated, both the prompt and some delayed $y$-rays are detected during the irradiation. Since every chemical element, except He, emits prompt $\mathrm{Y}$-rays, the method can in principle detect all elements in the periodic table, but with very different sensitivities. The most sensitive elements include B, Cd, Nd, Sm, Eu and $\mathrm{Gd}$, whereas the least sensitive elements are $\mathrm{Be}, \mathrm{C}$, $\mathrm{N}, \mathrm{O}, \mathrm{F}, \mathrm{Pb}$ and $\mathrm{Bi}$. Since the number of prompt $\mathrm{Y}$-rays is much higher compared to decay $\gamma$-rays, the prompt $y$-ray spectrum is generally much more complicated than the INAA spectrum. In both instances the identification of chemical elements and their quantitative analysis is based on the precise determination of energies and intensities of $\mathrm{Y}$-rays.

The first applications of PGAA were performed in Saclay and Grenoble in the late 1960s and early 1970s. This method has become more widely used since the 1980s, 


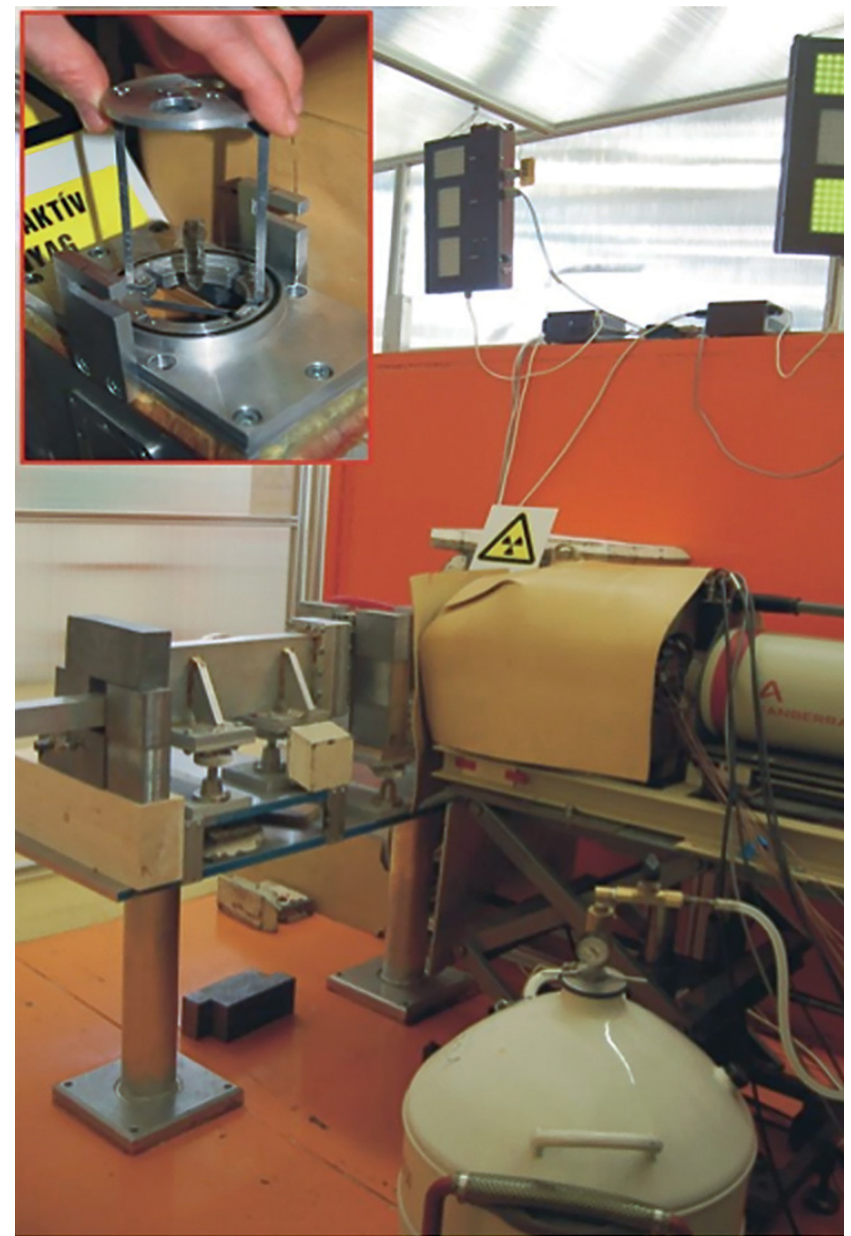

Figure 3.1: The Prompt Gamma Activation Analysis facility at the Budapest Neutron Centre. Insert photograph: A sample holder with a small obsidian sample.

when high intensity neutron guides with super mirrors were built next to research reactors and high resolution HPGe detectors became widely available. See Figure 3.1.

INAA induces at least some minimal damage to objects, but is very sensitive for trace element analysis. In contrast, intact objects remain after PGAA with almost no induced radioactivity, because of the lower $\left(10^{7}-10^{9} \mathrm{~cm}^{-2} \mathrm{~s}^{-1}\right)$ neutron flux in the beam.

Thus PGAA is more sensitive to major components and provides information on only a few trace elements with high neutron absorption cross sections $(\mathrm{H}, \mathrm{B}, \mathrm{Cl}, \mathrm{Cd}$ and rare-earth elements). For this reason, these two standard neutron activation techniques are complementary.

\section{NEUTRON DEPTH PROFILING (NDP)}

Neutron Depth Profiling is a prompt analysis technique which uses neutron-induced nuclear reactions accompanied by the emission of charged particles with a specific kinetic energy [96-98]. It is one of the most powerful nondestructive techniques for depth profiling of particular light elements, especially ${ }^{10} \mathrm{~B}$ and ${ }^{6} \mathrm{Li}$, which have very high thermal neutron capture cross-sections. The samples are placed in a thermal neutron beam (usually from a nuclear reactor) and the energy spectra of emitted charged particles are measured using semiconductor detectors (see figure 3.2 top). NDP is extensively used for the determination and depth profiling of ${ }^{3} \mathrm{He}\left(10^{-3}\right)$, ${ }^{6} \mathrm{Li}\left(10^{-3}\right),{ }^{10} \mathrm{~B}\left(10^{-2}\right),{ }^{14} \mathrm{~N}(1)$, and ${ }^{35} \mathrm{Cl}(10)$. The numbers in parentheses are typical detection limits in units of $10^{15}$ atoms $/ \mathrm{cm}^{2}$.

If the neutron-capture reaction takes place beneath the sample surface, the energy loss of the charged particles emerging from the sample can be used to obtain information about the element concentration profile as a function of depth (see figure 3.2 bottom). The magnitude of the energy loss is simply related to the distance that the charged particle has travelled within the specimen.
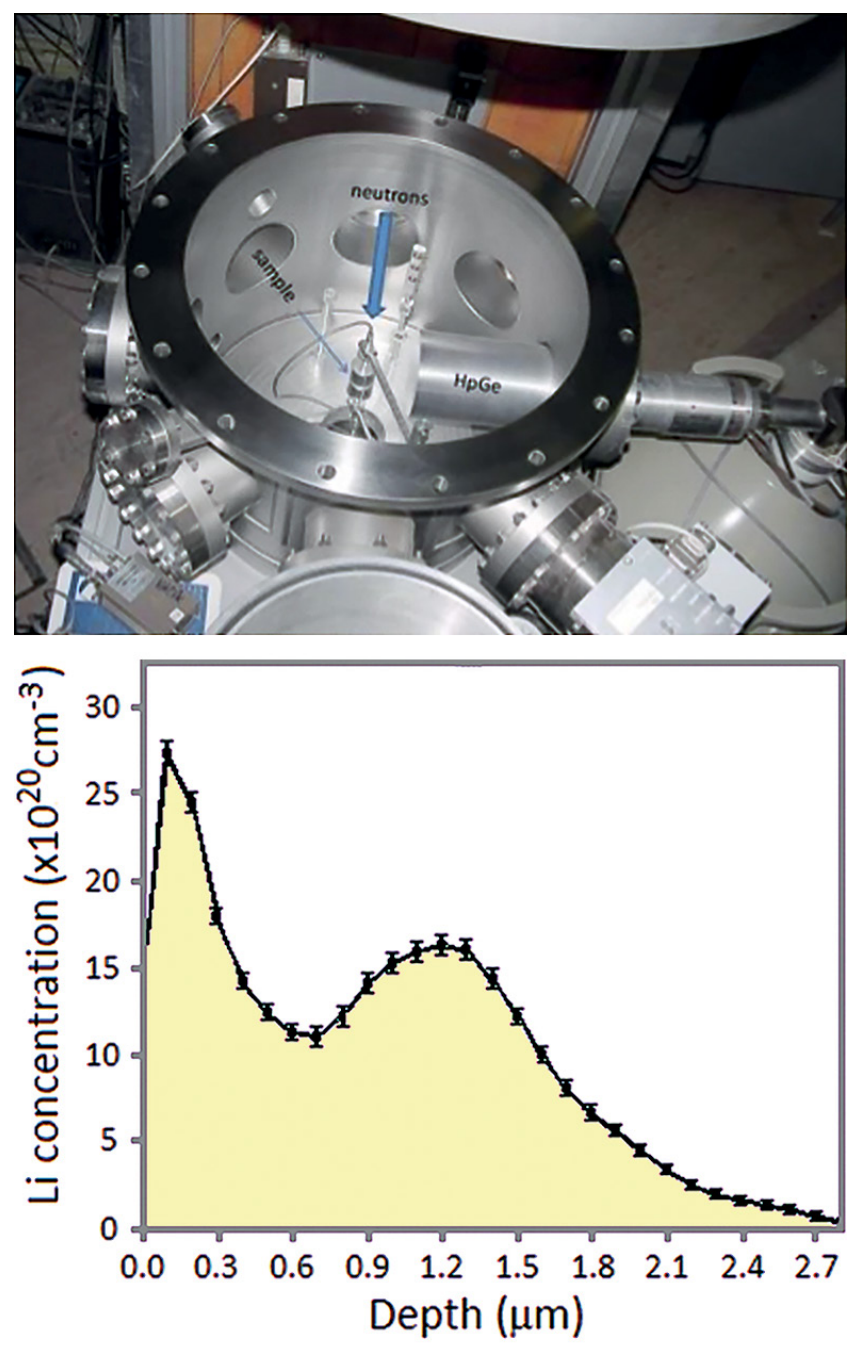

Figure 3.2: Top: Vacuum chamber for NDP analyses used by CANAM NPI at the nuclear reactor (Research Centre Řež Ltd.). Bottom: Depth profile of lithium atoms in tantalum determined from measurements of the energy spectrum of ${ }^{3} \mathrm{H}$ and ${ }^{4} \mathrm{He}$ particles emitted in the reaction ${ }^{6} \mathrm{Li}+\mathrm{n}$. The sample was implanted with $300 \mathrm{keV} \mathrm{Li}$ ions and annealed at $1800^{\circ} \mathrm{C}$. [97] 


\subsection{INSTRUMENTATION OF NEUTRON BEAMS}

Neutron beams are perfect tools for archaeometric studies. Various neutron techniques offer a wide range of methods of exploring the compositional or structural features of samples. Due to the low energy and relatively low intensity $\left(10^{7}-10^{9} \mathrm{~cm}^{-2} \mathrm{~s}^{-1}\right)$ of guided neutron beams, no damage to objects is observed, and the induced radioactivity decays within a few days.

Research reactors with compact cores, or acceleratorbased spallation sources, provide intense neutron beams for experiments. These are usually taken off from cold or thermal moderators by sophisticated neutron optical systems. These neutron beam transport devices are known as neutron guides - they provide collimated, highly parallel beams of thermal and cold neutrons for measuring instruments and spectrometers.

Based on different types of interactions of neutrons with matter, capture or scattering reactions can be used. For example, by detecting characteristic $y$-rays produced in $(n, y)$ reactions, the bulk elemental composition of objects can be determined. These techniques (NAA, PGAA, NDP) are described in detail in the previous section. On the other hand, through investigation of elastic or inelastic scattering patterns, information can be obtained on the atomic, molecular or nano-scale structural properties, including information on crystalline/ amorphous morphology, phase composition, mechanical strains, and impurities. The most important techniques and instrumentation relevant to investigations of cultural heritage objects are now described.

Neutron diffraction or elastic neutron scattering is the application of neutron scattering to the determination of the atomic or magnetic structure of a material. The technique is similar to $X$-ray diffraction but due to their different scattering properties, neutrons and X-rays provide complementary information: $X$-Rays are suited for superficial analysis; strong $x$-rays from synchrotron radiation are suited for shallow depths or thin specimens; neutrons, which have a high penetration depth, are suited for bulk samples.

Neutron diffraction is most commonly performed as powder diffraction, which only requires a polycrystalline powder or amorphous sample. The incoming neutron beam needs to be mono-chromatised, to sort the energies of the incident neutrons (higher energy neutrons are faster). This can be done using a single crystal monochromator. The time-of-flight (TOF) technique can also be used. No monochromator is needed in the TOF technique. Rather, a series of aperture elements (choppers) are synchronized to filter neutron pulses with the desired wavelength (energy). Neutrons coming from the sample are detected in counters to measure the angular distribution of the scattered neutrons. A typical example of such a diffractometer is in use at the Budapest Research Reactor.
This unique type of TOF powder diffractometer is used on a continuous beam chopped to produce several welldefined wavelength bands, which vary periodically within a selected wavelength range. This type of instrument usually outperforms a conventional crystal monochromator powder diffractometer and has increased resolution in atomic position determination. See Figure 3.3.

This TOF instrument plays an essential role in exploring the material properties of archaeological objects, e.g. crystalographic phase analysis can reveal ancient technologies. Differences in the diffraction patterns are usually due to different compositional and/or treatment features.

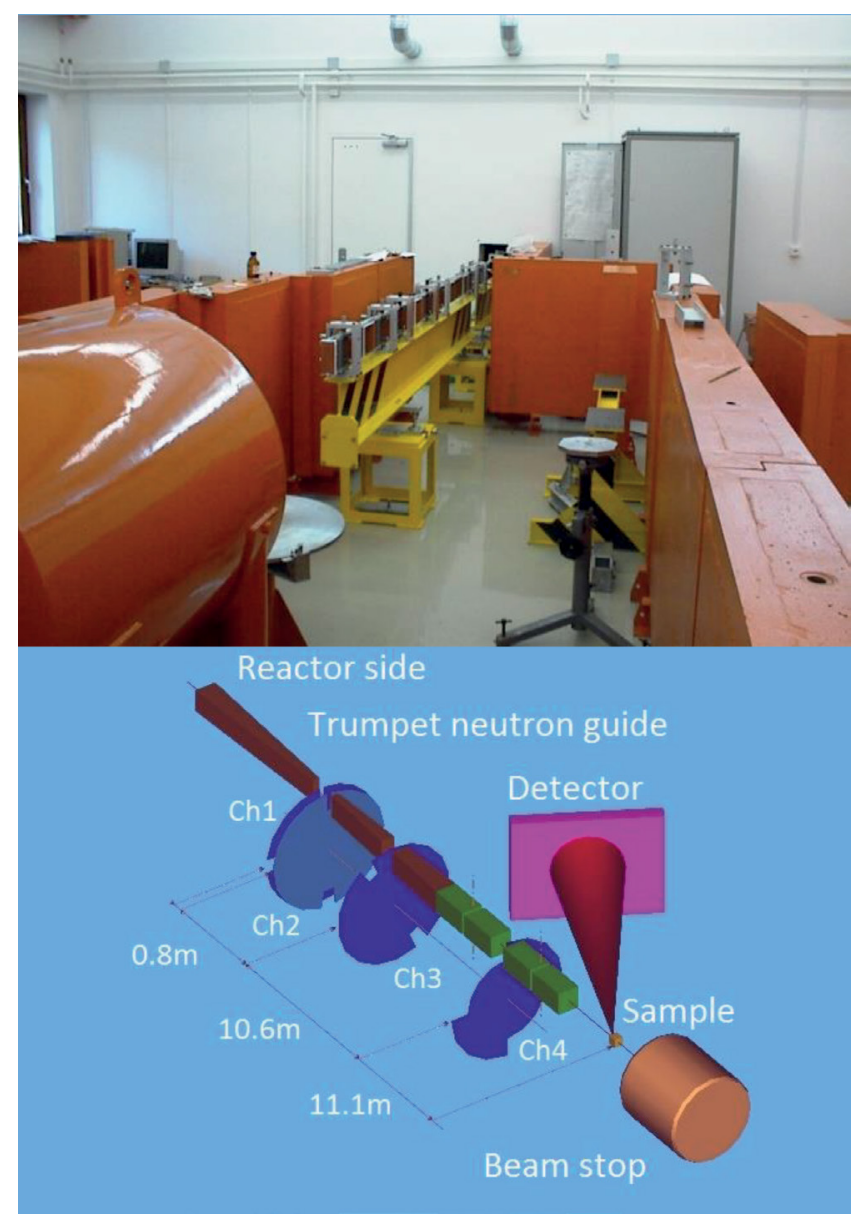

Figure 3.3: Photograph and Schematic illustration of the neutron TOF instrument set-up showing the real neutron guide together with its shielding components.

A typical application of this instrument is in the study of lapis lazuli, a beautiful light blue stone, which was a high grade gemstone and pigment in the Near-East, Egypt, and later in Europe. Its geological occurrences are well known and each have particular differences in their detailed chemical composition.

There were already attempts in antiquity to produce artificial lapis lazuli, such as Egyptian Blue, i.e. $\mathrm{CaCuSi}_{4} \mathrm{O}_{10}$. Both PGAA and time-of-flight neutron diffraction TOF-ND are now capable of distinguishing between false and true lapis lazuli [94]. 


\section{SMALL ANGLE NEUTRON SCATTERING (SANS)}

SANS is a technique for studying nanometer scale structural features in materials. The information obtained, however, is characteristic of the whole irradiated volume of the sample.

Practically, the measured piece is free from deformation, physical, chemical or structural changes and there is no need for sampling.

The SANS technique can determine void sizes in porous media such as cements and marble. It can also identify anisotropies in precipitate orientation in minerals or metals and facilitates the investigation of particle agglomeration in ceramic bodies and the evolution of pores during different types of processing.

In the majority of SANS experiments the scattering of neutrons is isotropic; the studied particles can be regarded as spheres. So far anisotropic SANS experiments were mostly used to study the shape and orientation of defect agglomeration in single crystals based on the asymptotic behaviour of the scattering pattern. However 2D data analysis software has been developed and successfully used for non-isotropic SANS data.

The Yellow Submarine SANS spectrometer, operating at the cold neutron source of the Budapest Research Reactor, is a large-scale instrument that covers a study range of material inhomogeneities from $50 \AA$ to $1500 \AA$.

SANS can be a proper tool for investigation of structural differences between marble, ceramic, metal objects of different provenance or technologies.
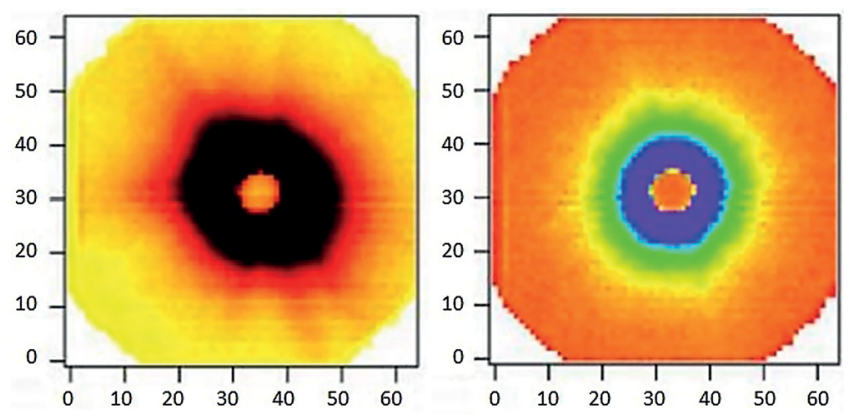

Figure 3.4: Comparison betwen two 2D neutron scattering patterns, measured using a $64 \times 64$ pixel position sensitive neutron detector, from two different marble samples.

A study on archaeological marble samples is presented in Figure 3.4. The left sample shows a strong anisotropy, which is caused by the morphology of the marble as a consequence of its geological history, while the right sample is isotropic.

\section{NEUTRON DEPTH PROFILING (NDP)}

Samples are irradiated with thermal neutrons and the energy spectra of charged particles are measured by semiconductor detectors (PIPS, PINs) connected to standard electronic devices for data acquisition [99]. The samples are irradiated in a vacuum chamber placed on external neutron beam from a nuclear reactor. The neutron beam is usually transported to the chamber via a neutron guide, transparent for thermal neutrons. The size of the neutron beam can be limited by collimators of neutron absorbing materials. The beam size can further be reduced, down to micrometer size, by neutron lenses constructed from glass capillaries.

\subsection{APPLICATIONS OF NEUTRON BEAMS}

The great advantage of neutron-based methods in researching Cultural Heritage objects is that the majority of techniques are completely nondestructive and non-invasive, although some may require a small sample to be extracted discretely from the object. Generally this means that no visible modifications are observed following investigations. Information on both the composition and structure can be obtained from priceless artefacts made of rocks, metals, ceramics, glass, etc. The combination of neutronbased techniques with other methods, such as PIXE, can provide even more comprehensive and valuable information.

\subsubsection{PROVENANCE OF PREHISTORIC STONE TOOLS}

In prehistoric times people knew where good quality materials could be quarried to make everyday tools. Sometimes, final or semi-final products were carried hundreds of kilometres from the localities where the raw materials were mined. If we can analyse the composition of tools and fingerprint chemical components characteristic of the material's provenance, this can enormously help archaeologists to reconstruct prehistoric trade and migration routes.

Non-destructive studies can also help distinguish between different basic types of raw materials (e.g. obsidian, flint, silex, felsitic porphyry) which are sometimes easy to confuse on the basis of visual examination [100]. In the Budapest PGAA laboratory, studies have found that knowledge of the bulk elemental composition can differentiate between obsidian, silex and felsitic porphyry.

The easiest task is to determine the provenance of obsidian - a volcanic glass which was popular as a raw material from the early Palaeolithic period. See Figure 3.5. The geological sources are quite well known and their compositions are distinctive of the geological formation and thus define the locality. Besides trace elements of $R b$, $\mathrm{Nb}, \mathrm{Yb}$, etc. that can be measured by destructive INAA, B and $\mathrm{Cl}$, which are both easy to measure non-destructively by PGAA, were found to be fingerprints as well. 
Using a PGAA obsidian database, a better overview of the distribution of obsidian material in Central Europe has been obtained. In particular a border zone between the distribution areas of the so-called Carpathian and Lipari obsidians has been identified. This border falls in the inland part of modern Croatia [101].

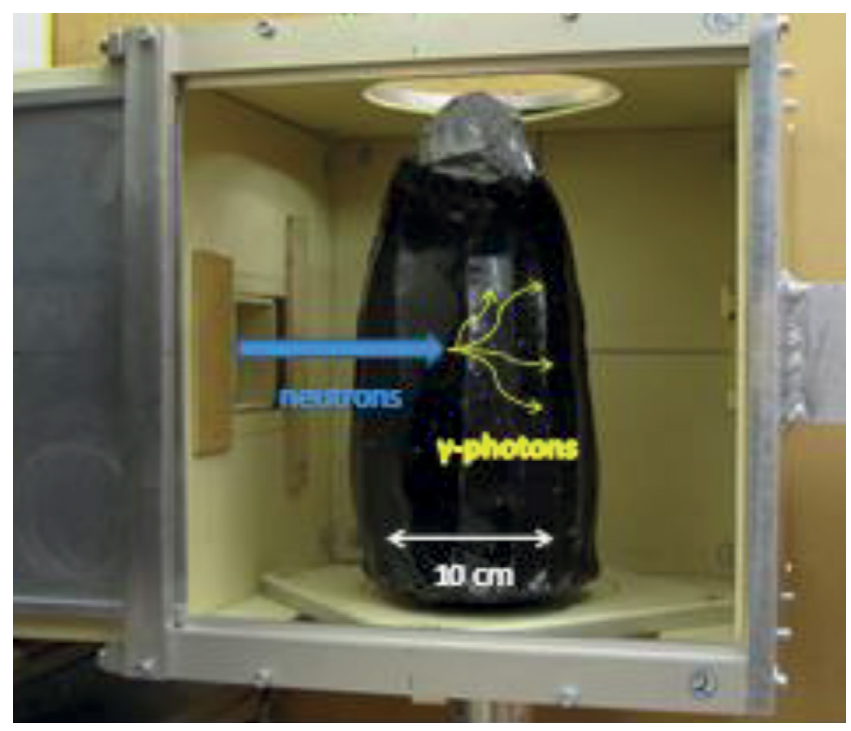

Figure 3.5: An obsidian core from Nyirlugos, Hungary held in the sample holder of the NIPS (PGAA) station of the Budapest Neutron Centre. The object is from the collection of the Hungarian National Museum.

In cases where it is allowed to take samples from the objects, INAA could help to quantify additional trace elements. With an extended database including both major and trace components, one can try to perform provenance analysis. In the investigation of stone objects the PGAA method has a great advantage when it is necessary to determine the average bulk composition of a rock, without sampling any unique objects.

\subsubsection{TRACING ANCIENT POTTERY}

From Neolithic times, ceramics have formed the most abundant group of archaeological discoveries. Various styles, production techniques and workshops can be identified on the basis of typology, as well as using analytical methods. The results can provide information about the exchange of goods and the spread of techniques, etc.

Since ceramic is a composite material consisting of clay, temper and sometimes glaze or paint layers on the surface, one has to be careful choosing the most appropriate method and properly interpreting the results.

From the chemical composition of clay, one can identify deposits where the raw material might originate. Major clay components can be determined using PGAA (in fact an average composition is determined for just a few $\mathrm{cm}^{3}$ ). Characteristic trace elements can be determined with the help of INAA or X-rays. Further microscopic studies of thin sections can help to identify workshops or cultures.
A project lead by the Simon Bolivar University in Caracas, Venezuela, aimed to identify the raw material used to make pre-Columbian $\left(13^{\text {th }}-15^{\text {th }}\right.$ c. $\left.A D\right)$ pottery figurines made in the Valencia Lake Basin region [102]. Fragments from the continental (Valencia Lake Basin) as well as from the nearby Caribbean islands (Los Roques Archipelago) have been analysed by PGAA and by INAA. As a result we have found, with high confidence, that inhabitants of the Los Roques Archipelago used local clay to produce the figurines instead of importing the raw material from the continent. See Figure 3.6.

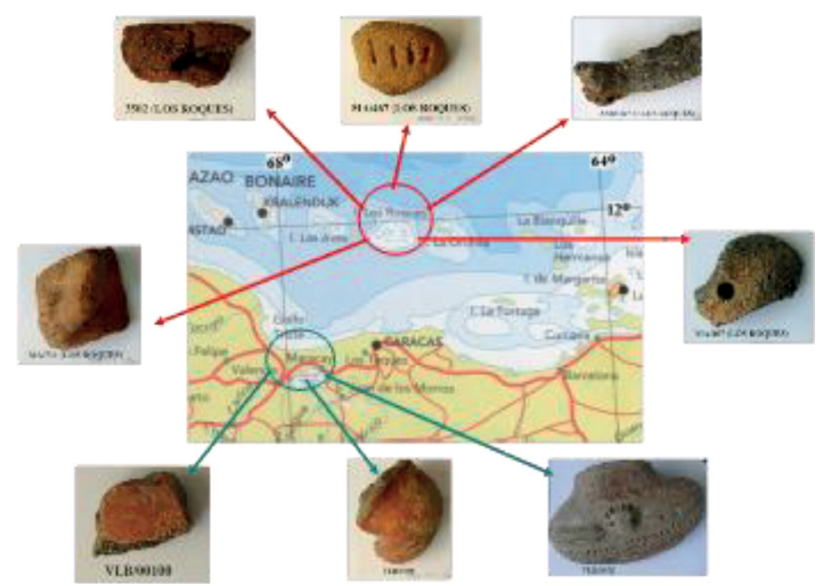

Figure 3.6: Origin of pre-Columbian pottery fragments studied by PGAA at the Budapest Neutron Centre.

PGAA and NAA have been applied in many research projects on archaeological ceramics from Europe [103], as well as from the New World [102,104]. Besides the chemical composition, the structure of ceramic materials on a micro or nanoscale may deliver information about the characteristics of production (fineness of clay, firing temperatures, etc.). For this purposes, TOF-ND and Small Angle Neutron Scattering (SANS) can be used [105].

\subsubsection{DEVALUATION OF NOBLE METALS}

Silver and gold have been some of the most valuable materials in history from ancient Egypt ( $3^{\text {rd }}$ millennium B.C.) up to present times. Since their value can be considered relatively constant with time, silver and gold functioned as currency. The production of silver from silver-bearing minerals (argentite $-\mathrm{Ag}_{2} \mathrm{~S}$ or galenite - PbS with $1 \%$ $\mathrm{Ag}_{2} \mathrm{~S}$ ) was first carried out in ancient Anatolia. However, during the time of the Roman Empire, the values of coins were intentionally modified by different Emperors. In the course of an economic crisis, the silver content of coins was gradually reduced. Interestingly, this was done without any visible sign on the coin surfaces.

Thanks to non-invasive PGAA, it was possible to show that the total silver content of later coins were significantly lower. On the other hand, it was impossible to detect this tendency, either by visual observation or by surface sensitive XRF analysis [106]. 


\subsubsection{METEORIC JEWELLERY}

A study combining various neutron beam methods has revealed that 5000 year old Egyptian iron beads have been found to be made from hammered pieces of meteorites [107]. The study focuses on the earliest known iron artefacts - nine small beads securely dated to circa 3200 BC, from two burials in Gerzeh, northern Egypt. The iron beads were strung into a necklace together with other exotic minerals such as lapis lazuli, gold and carnelian, revealing the status of meteoritic iron as a special material on a par with precious metal and gem stones. See Figures 3.7 and 3.8.

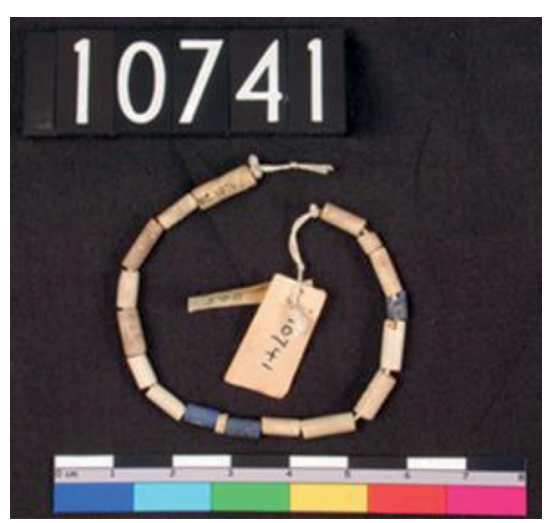

Figure 3.7:

Collection of

stone and faience beads from Tomb 67. Modern restringing, without the iron beads.

Figure 3.8: Neutron radiographs of the three beads 10740 , 10739, 10738 (from top), in side view and longitudinal.
The experimental study of three iron beads was performed at the Budapest Neutron Centre. Neutron and proton beam techniques were applied: PGAA, TOF neutron diffraction and PIXE measurements provided compositional data showing that the beads were made from meteoritic iron.
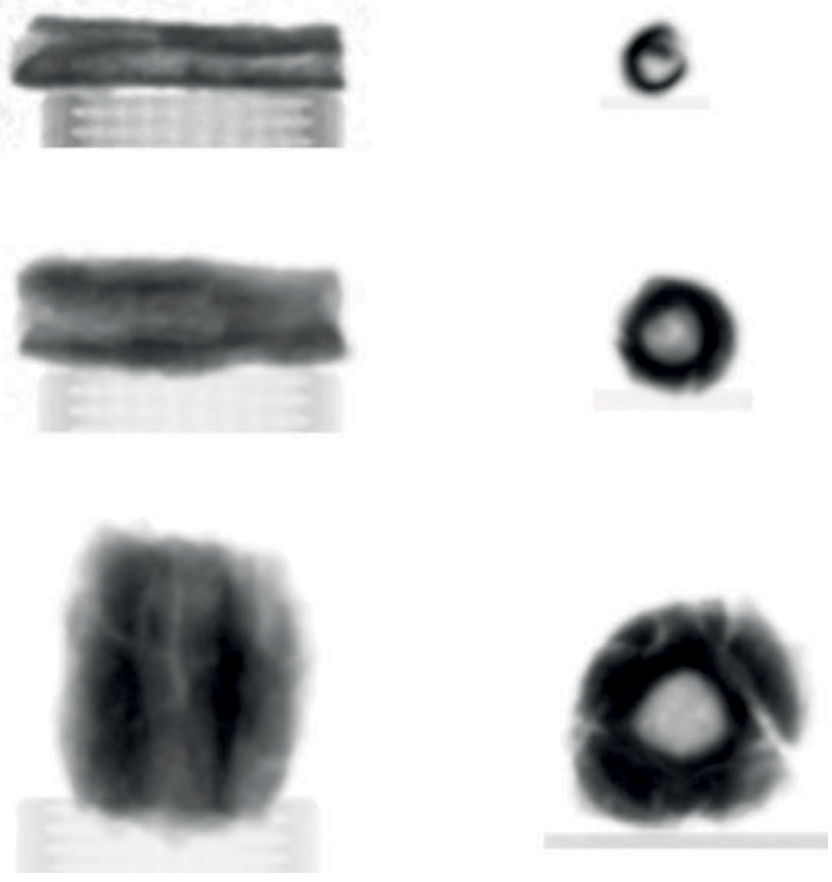

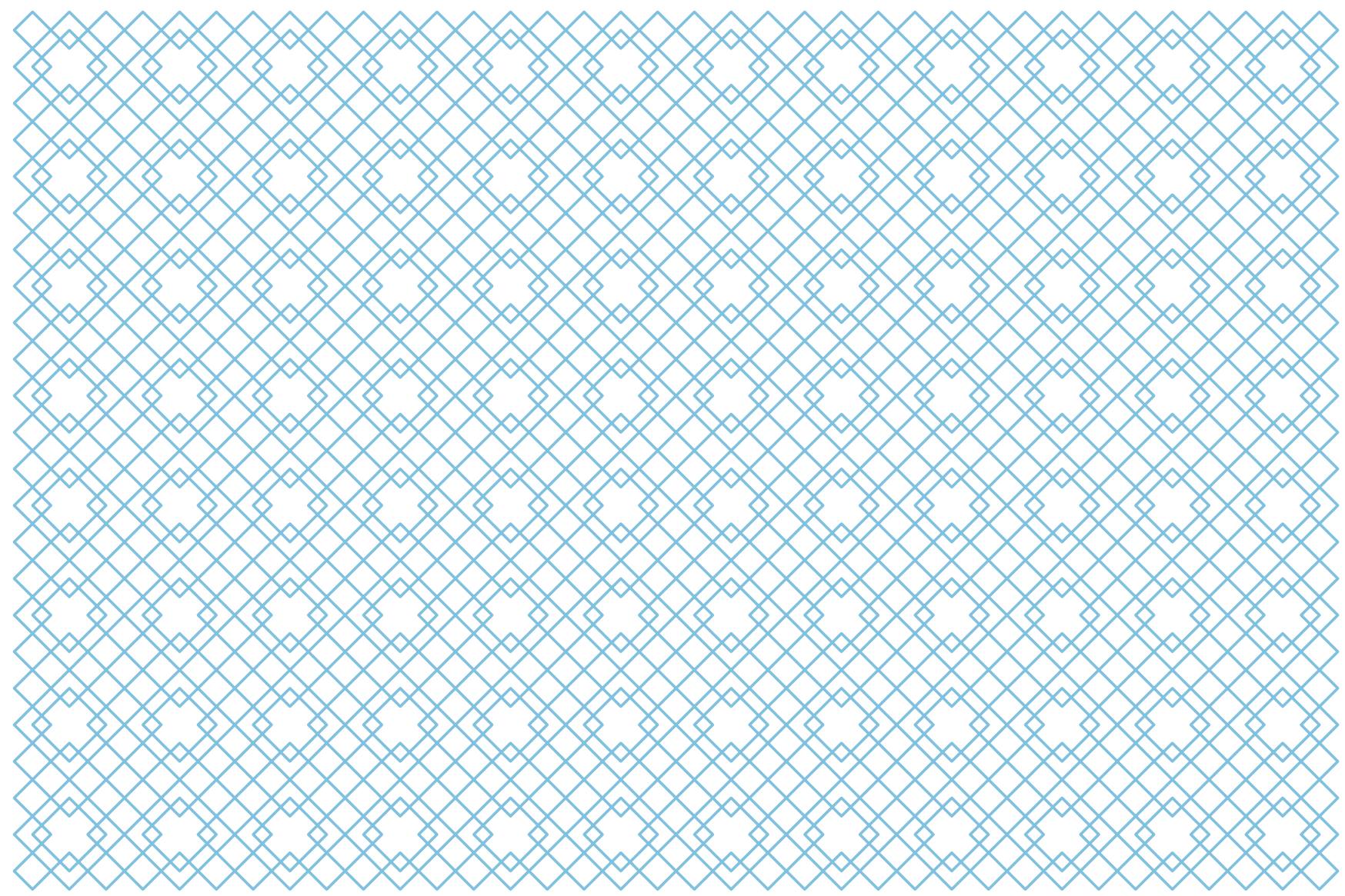

